\title{
A Coordinate System for the Synthesis of Visual and Kinesthetic Information
}

\author{
S. I. Helms Tillery, M. Flanders, and J. F. Soechting \\ Department of Physiology, University of Minnesota, Minneapolis, Minnesota 55455
}

\begin{abstract}
The results of this study suggest that information derived from kinesthetic inputs alone is not normally used to generate an estimate of the location of the hand in extrapersonal space. This finding provides support for the interpretation of previous results suggesting that a representation of a visual target in extrapersonal space must be transformed into a kinesthetic reference frame before the parameters of a targeted arm movement can be computed (Soechting and Flanders, 1989b). We asked subjects to use a pointer to indicate the spatial location of their hand following an unseen passive displacement. We found that subjects had large errors in the locations that they chose and that there was a large degree of variability for repeated trials with the same hand location. The errors were a result neither of using the pointer to indicate a spatial locus nor of an inability to make use of kinesthetic information. Instead, the errors resulted from an inability of subjects to synthesize an estimate of the hand's spatial location from only kinesthetic cues. We also asked subjects to use the pointer to indicate the location of their hand following a passive displacement when they had visual information about the passive displacement. In this case, we found that subjects performed better than when they had only kinesthetic information, but not as well as when they had only visual information about target location. This finding suggests that kinesthetic information about target location affects the processing of visual information.
\end{abstract}

In order to understand the generation of arm movements, it is important to establish the types of computations involved in specifying movement parameters and to identify the coordinate frames in which those computations are carried out. When information from several sensory modalities is to be involved in those computations, it becomes important to put information from different modalities into a common coordinate frame. One possible use that can then be made of these sources of information is a comparison between the goal of an arm movement and the current state of the arm. For the simple task of moving to a stationary target, this computation of "motor error" may be a comparison between the current end point of the arm (the hand) in space and the desired end point. In fact, data from the primary motor cortex suggest that motor error is encoded as the desired direction of the hand's movement in space (Geor-

\footnotetext{
Received June 6, 1990; revised Sept. 17, 1990; accepted Oct. 23, 1990.

This paper is based upon work supported under a National Science Foundation Graduate Fellowship and by NIH Grants NS-15018 and NS-27484.

Correspondence should be addressed to S. I. Helms Tillery, Department of Physiology, 6-255 Millard Hall, University of Minnesota, Minneapolis, MN 55455.

Copyright (C) 1991 Society for Neuroscience $0270-6474 / 91 / 110770-09 \$ 03.00 / 0$
}

gopoulos et al., 1988). This hand-centered representation of movement direction provides a constraint on the computation of motor error that leads to arm movement.

In the case where the hand is not in view at the initiation of the movement, the location of the hand must be given by kinesthetic inputs. These inputs, however, are encoded in a reference frame quite distinct from the reference frame in which the visual target is initially encoded. The question then arises: In what reference frame are the current and desired hand locations compared?

We have used a simple task to study the computations that lead to the final representation of movement parameters (Soechting and Flanders, 1989a, b; Flanders and Soechting, 1990; Soechting et al., 1990). The task is a movement of the arm to the remembered location of a visually specified target (a virtual target). In most cases, the movements were performed in the dark. Therefore, if sensory information about arm position were to be used in the computation of movement parameters, that information would likely come from kinesthesis. The initial finding of the experiments was that the desired position of the arm, described as the arm segment orientation angles, was determined by a simple set of linear relations to target location, as specified in spherical coordinates from the shoulder (Soechting and Flanders, 1989b).

In order to explain this finding and place it into the context of existing neurophysiological data, we proposed a model of the computational steps involved (Soechting and Flanders, 1990). The basic elements of the model are stages that convert a target, specified initially by the visual system, to a motor error signal consistent with the tuning characteristics of motor cortical neurons. Specifically, a target that is initially represented in a retinotopic frame is stabilized to a head-centered frame of reference and then shifted to a shoulder-centered frame of reference. The shoulder-centered target representation is then transformed into a set of desired arm orientation angles. According to the model, this set of desired orientation angles is compared with the actual arm orientation, giving rise to the motor error signal. In other words, the model postulates that the target representation is converted into a coordinate frame identical to the coordinate frame for the encoding of kinesthetic parameters, and in this reference frame, the motor error is computed.

Assuming that motor error is computed in a common frame, there is at least one obvious alternative to the use of arm segment orientations. Instead of computations performed in a kinesthetic reference frame (arm orientation), it is possible that kinesthetic information is converted into a reference frame more similar to the visual reference frame in which target location is initially represented (position of the hand in space). In this case, motor error could be computed in a spatial reference frame. If this 
were the case, then it should be possiblc to synthesize an estimate of the spatial location of the hand using only kinesthetic cues. In the experiments described in this paper, we examined this question by asking subjects to estimate the locations of their hand following passive displacements of the arm.

Because kinesthetic inputs provide information more directly related to the orientation of the arm (joint angles, muscle length, etc.), the end point (hand location) of the arm would need to be computed. We hypothesized that subjects might estimate the location of their hand in space using a mapping from a coordinate system based on the orientation of the arms to a coordinate system based on locations in extrapersonal space. We also hypothesized that subjects would compute the end point of the arm by reversing the linear transformation used to compute a desired arm orientation from a shoulder-centered target representation (Soechting and Flanders, 1989b). Contrary to these hypothcscs, we found that subjects were unable to synthesize a reliable estimate of the locations of their hands in space using only kinesthetic cues, and that the estimates that they provided were not reversals of the previously described transformation. We also found that they were able to reliably reproduce the arm segment orientations of their passively displaced arms. These results provide support for the computational model described above.

\section{Materials and Methods}

\section{Tasks}

We presented subjects with targets at random locations throughout their workspace. The targets were either presented visually by the experimenter or kinesthetically by passively displacing the subjects' arms. After a brief interval, the subjects were asked to perform a movement to indicate the target's remembered location. "Target end point" was defined as either the location of the index finger in the passive displacements or the location of the visually presented target. The "movement end point" was taken to be either the end of a pointer used by the subjects to indicate the target location or the location of the index finger after an active arm movement.

There were 4 experimental conditions:

Match passive (MP). This experiment was designed to assess the ability of subjects to make use of kinesthetic information arising from passively imposed movements. ${ }^{1}$ The experimenter passively displaced the subject's right arm in the dark. After being held briefly in position, the arm was returned to the subject's side. The subject was then asked to move the arm to the same target end point. No further instructions were given as to the aspect of the passive displacement that the subjects were supposed to reproduce.

Two alternative strategies exist for the performance of this task: Subjects could attempt to reproduce the end point or to match the arm orientations of the passive displacement. To distinguish between these 2 possibilities, we performed the following experiment in which the subjects were forced to match the end point of the passive displacement alone.

Match end point, pointer; dark ( $M E, d k)$. This experiment was designed to determine whether subjects were able to estimate their hand's location in extrapersonal space based upon kinesthetic information. The experimenter passively displaced the subject's right arm in the dark. After returning the arm to the rest position, the lights were turned on, and the subject was asked to indicate the end point of the passive movement with the tip of a pointer (about $1 \mathrm{~m}$ in length) that was grasped in the

\footnotetext{
' It has been shown that subjects perform better in matching paradigms when target movements are actively performed than when the target movements are passively imposed (see especially Paillard and Brouchon, 1968). We used the MP experiment as a control that allowed us to make all of our comparisons between passive conditions. While there may be improved performance on the tasks under active conditions, there is no reason to expect that those improvements would be substantially greater for the ME,dk task than for the MP task.
}

right hand. The subjects were given no further instructions as to the use of the pointer and were free to use any movements they felt necessary to place the tip of the pointer at the target location (including adjusting the location of their grasp on the pointer).

Virtual target, pointer $(V T)$. The following control experiment was performed to test the reliability with which the subjects could indicate a visually specified target in space using the pointer. (We had previously determined the reliability with which subjects could indicate a point in space using the right arm; Soechting and Flanders, 1989a.) A visual target, which was a small stylus held by the experimenter, was briefly presented to the subject. The subject was then asked to use the pointer to indicate the location of the target.

Match end point, pointer; light (ME,It). To see whether vision or kinesthesia predominated in determining the location of the hand in space, the ME,dk experiment was repeated, but with the lights turned on throughout the experiment so that the subjects could see the location of their passively displaced hands.

\section{Data acquisition}

At least 70 trials were given to each subject under each condition, with the target locations varying randomly from trial to trial. Five subjects participated in the study, with 4 subjects being used for each of the conditions reported here. Target location and arm orientation were monitored with an ultrasonic system (Graf-Pen GP8-3D, Science Accessories). The source for the ultrasound was a small stylus-shaped emitter. Emitters were located at the shoulder, elbow, and wrist to monitor arm position. In addition, a fourth emitter was held either by the experimenter during the presentation of visual targets or by the subject during the presentation of kinesthetic targets and during the movements. The location of the emitters was monitored by 4 microphones situated on the corners of a $1.5 \times 1.5 \mathrm{~m}$ square. Time for the sound to reach each of the microphones was converted to distance, and the location of each of the emitters was calculated trigonometrically. With this system, the location of each stylus could be determined at a resolution of $0.1 \mathrm{~mm}$ anywhere within a cube $\sim 1.5 \mathrm{~m}$ on a side.

\section{Data analysis}

Arm orientations were represented as shown in Figure $1 A$. The coordinate system shown has been demonstrated psychophysically to be the preferred one for the perception of arm orientation (Soechting and Ross, 1984). The 4 angles are the elevation (angle relative to a vertical axis) of the upper $(\theta)$ and lower $(\beta)$ arms and yaw (angle relative to a sagittal plane) of the upper $(\eta)$ and lower $(\alpha)$ arms.

Target and movement end-point locations were represented in spherical coordinates relative to the shoulder (Fig. $1 B$ ). In this case, the parameters are distance $(R)$, azimuth $(\chi$, angle relative to a sagittal plane), and elevation ( $\psi$, angle relative to a horizontal plane).

End-point data were analyzed with multivariate linear regression methods as described by Johnson and Wichern (1982). Linear, quadratic, and cubic equations were constructed to describe each of the parameters of the subjects' movements as a function of the target parameters. The analysis led to equations of the following form, where a $T$ subscript indicates a target parameter and an $M$ subscript indicates a movement parameter:

$$
\begin{array}{rlr}
X_{M}= & a_{0}+a_{1} R_{T}+a_{2} \chi_{T}+a_{3} \psi_{T} \\
X_{M}= & \text { (linear form) }+b_{4} R_{T}{ }^{2}+b_{5} R_{T} \chi_{T}+\cdots \\
& +b_{9} \psi_{T}^{2} & \text { (linear) }, \\
X_{M}= & \text { (quadratic form) }+c_{10} R_{T}^{3}+c_{11} R_{T}{ }^{2} \chi_{T} \\
& +\cdots+c_{19} R_{T} \chi_{T} \psi_{T}
\end{array}
$$

These equations were generated for $R_{M}, \chi_{M}$, and $\psi_{M}$. The $95 \%$ confidence intervals were computed for each of the 20 possible parameters in the cubic equations. Those parameters for which the interval did not include 0 were used to describe the performance of the subjects and are referred to as the "best-fit equations."

To test the hypothesis that subjects performed the MP task by matching the arm orientation angles of the passive displacement, regression analyses were also performed on the arm orientation angles using the singular value decomposition method as described by Press et al. (1988). The orientation angles chosen by the subject were related to the angles 
A

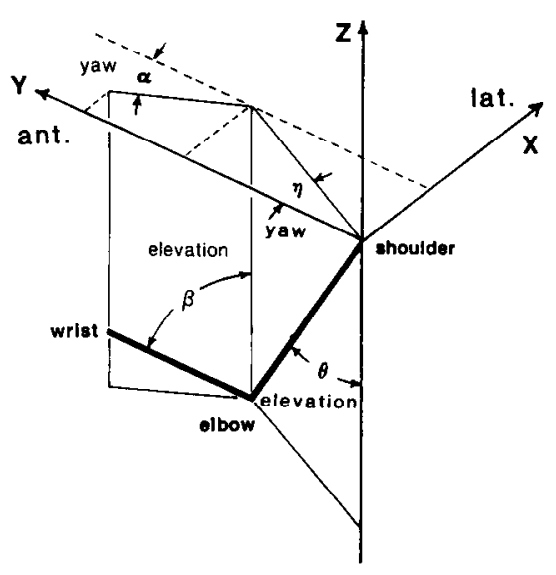

B

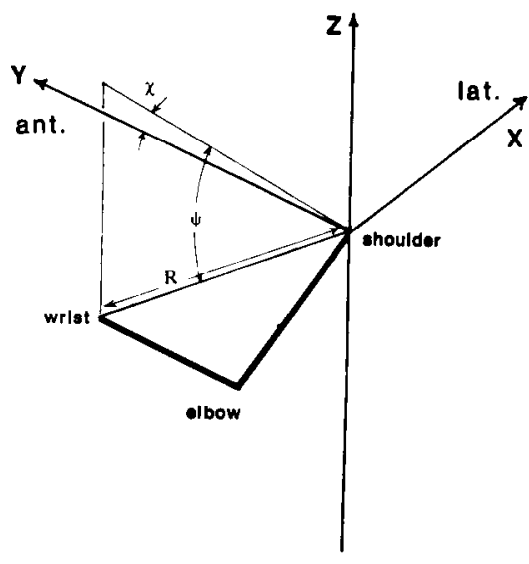

in the passively displaced arm. This analysis resulted in equations of the following form:

$$
\begin{array}{lr}
X_{M}=a_{0}+a_{1} \eta_{T}+a_{2} \theta_{T}+a_{3} \alpha_{T}+a_{4} \beta_{T} & \text { (linear), } \\
X_{1}=\text { (linear form) }+b_{5} \eta_{T}{ }^{2}+\cdots+b_{14} \beta_{T}{ }^{2} & \text { (quadratic), (2) } \\
X_{M}=\text { (quadratic form) }+c_{15} \eta_{T}{ }^{3}+\cdots+c_{34} \beta_{T}{ }^{3} & \text { (cubic). }
\end{array}
$$

In this case, $X$ represents 1 of the 4 arm orientation parameters $\left(\eta_{M}, \theta_{M}\right.$, $\alpha_{M}, \beta_{M}$ ). Again, best-fit equations were constructed, as described above, that contained only those parameters that contributed significantly to the fit.

As discussed earlier, when subjects are asked to move their arms to a visually presented target, the orientation that they choose for their arm segments is given by a simple linear combination of the parameters defining target location relative to the shoulder (Soechting and Flanders, $1989 \mathrm{~b}$ ). One possibility in performing the ME, dk task is that the subjects would simply reverse that linear transformation to give a spatial representation of their hand's location by a simple combination of the parameters defining arm orientation. To test this possibility, we also performed regression analyses to relate the end point chosen by the subjects in the ME, $\mathrm{dk}$ condition to the orientation angles of the passively displaced arm. This resultcd in cquations of the same form as Equation (2), but instead gave the end-point parameters chosen by the subject in terms of the passively displaced segment orientation angles.

These regression analyses were performed on the data from each of the subjects. In addition, in order to identify performance characteristics common across the subjects, we pooled the data from all the subjects under each condition and performed regressions on these pooled data.

The results of the analyses are presented in 2 ways. The best-fit equations are used to present the consistent aspects of the subjects' performance, and the difference between these equations and perfect performance will be referred to as "constant error." This term is chosen to reflect the fact that any consistent errors made by the subjects are described by the regression equations. To establish the variability in performance, variable error $\left(E_{x}\right)$ was computed for each parameter as follows:

$$
E_{X}^{2}=\frac{1}{n} \sum_{i=1}^{n}\left(X_{\text {data } . i}-X_{\text {fit. } i}\right)^{2}
$$

where $X$ is either an end-point parameter or an arm orientation angle. In addition, a total variable error $\left(E_{\mathrm{Tot}}\right)$ was computed for the end points:

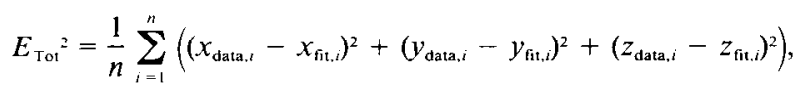

where $x, y$, and $z$ are end-point locations in Cartesian coordinates. $E_{\mathrm{T} o 1}$ provides a measure of the mean distance between the locations chosen by the subject and the locations predicted by the fit.

\section{Results}

Two aspects of performance will be presented in this paper: constant error and variable error. As discussed in Materials and Methods, constant error represents the aspects of performance that were consistent from trial to trial, and variable error is a measure of the random variability in performance from trial to trial. Two central findings are reported in this paper: (1) The performance in the MP condition could be accounted for by assuming that the subjects attempted to match the arm orientation of the passive displacement rather than its end point, and (2) that the subjects were unable to reliably identify the spatial location of their hands based on kinesthetic cues alone.

\section{Variable error}

The variable error is presented in Figure 2. For each subject and experimental condition, we computed the variable error using Equation 3. In Figure 2, we show the average value of this measure for the 4 subjects. We did an ANOVA across these 4 conditions plus a fifth condition, which was a pointing movement of the arm to a visually presented target. The results showed significant effects $(p<0.01)$ in all of the measures of variable error shown in Figure $2: E_{R}, F(4,19)=7.56 ; E_{x}, F(4,19)=$ $4.97 ; E_{\psi}, F(4,19)=9.73 ; E_{\text {Tot }}, F(4,19)=8.20$. Multiple comparisons were performed with the Bonferroni $t$ test, with $t$ adjusted for the number of comparisons, and are reported as significant if conditions for $p<0.05$ were satisfied.

Our experimental conditions required subjects to use 2 sensory modalities to locate the target: visual (VT), kinesthetic (ME,dk and MP), or both (ME,lt). Two different kinds of movements were used to assess performance: the arm (MP) or a pointer (VT and ME). Variable errors for the movement of a pointer to a virtual target (VT) were comparable to those found previously when subjects used arm movements to indicate virtual targets (Soechting and Flanders, 1989a). Therefore, irrespective of whether subjects used a pointer or their arms, variable errors in different experimental conditions can be used to assess the ability to synthcsize the location of a point in space using visual or kinesthetic information.

For the total variable error (root mean square distance between locations predicted by best-fit equations and locations actually chosen by the subject), the only significant differences 

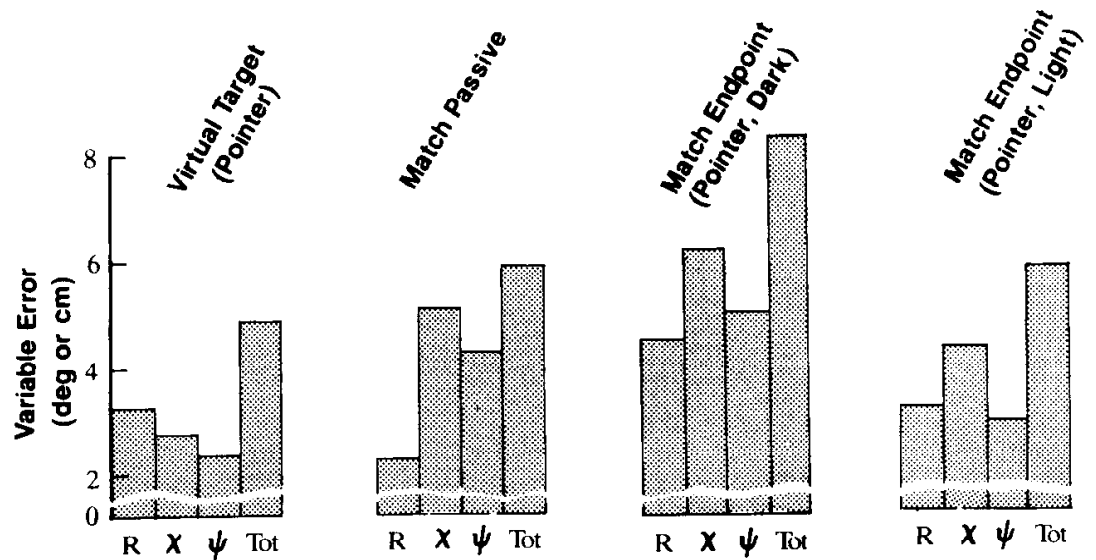

$\mathrm{R} \times \boldsymbol{\psi} \mathrm{Tot}$
Figure 2. Variable errors for 4 experimental conditions. Variability was greatest when subjects attempted to estimate their hands' locations given only kinesthetic information (ME,dk). Each bar gives the mean of the values for 4 subjects. found were between the ME, dk and the 4 other conditions (VT, MP, and ME,lt, as shown in Fig. 2, and also a virtual target, arm condition, which is not shown). The variable error in elevation was higher for both the ME,dk and the MP conditions than for the VT condition and was higher for ME,dk than for $\mathrm{ME}$,lt. The variabie errors for distance were higher for the ME,dk condition than for the MP condition. Variable errors in azimuth were higher for the ME,dk condition than for both the VT condition and the virtual target, arm condition (not shown). When asked to indicate the location in space of their hands based only on kinesthetic cues (ME,dk), the performance of the subjects showed a greater degree of variability from trial to trial than in any of the other conditions.

\section{Matching passive movement}

The constant error in the passive matching condition is shown for the 3 end-point parameters in Figure 3. It was computed using data pooled from all 4 subjects. The horizontal axes of the plots are the target parameters. The vertical axes give the movement parameter values predicted by the best-fit equations for the individual trials, and the deviation from the $45^{\circ}$ line represents constant error. Three aspects of the data stand out: First, the relations between the target parameters and the location predicted by the best-fit equations were close to linear. Second, the data were very close the $45^{\circ}$ lines on these plots over most of the range of target locations. Last, the vertical scatter in Figure 3 is quite small (e.g., cf. Fig. 6). (Vertical scatter on these plots results when one of the movement parameters is reliably determined by more than 1 parameter describing target location, e.g., when the movement elevation chosen depends on the target's azimuth and/or distance as well as on the target elevation). Consistent with these 3 aspects of the plots, the mean distance between the locations predicted by the best-fit equations and the target positions was low $(4.75 \pm 0.66 \mathrm{~cm}$, mean \pm SE for 4 subjects, compared to $5.62 \pm 0.97 \mathrm{~cm}$ for the VT condition) and did not depend on the location in the workspace.

In Figure 4, we show the results for the same set of experiments when the pooled data are represented in arm orientation space. In this case, the orientation parameters showed a greater degree of scatter about the $45^{\circ}$ line. Each of the parameters

\section{Match Passive, Endpoint Parameters}
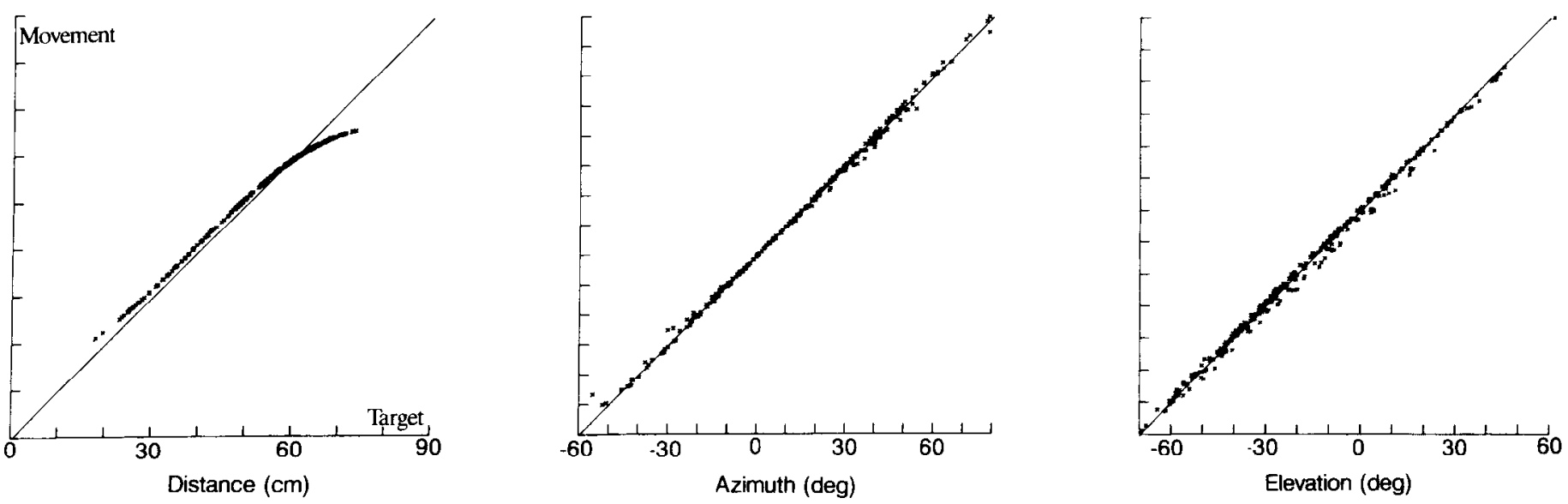

Figure 3. Constant error for the MP condition represented in end-point coordinates for pooled data from 4 subjects. Performance in this condition was close to linear and did not change across the work space. Movement parameters are plotted as a function of target parameters, and the data points are the locations predicted by the best-fit equations for each parameter. 


\section{Match Passive, Arm Orientation Parameters}

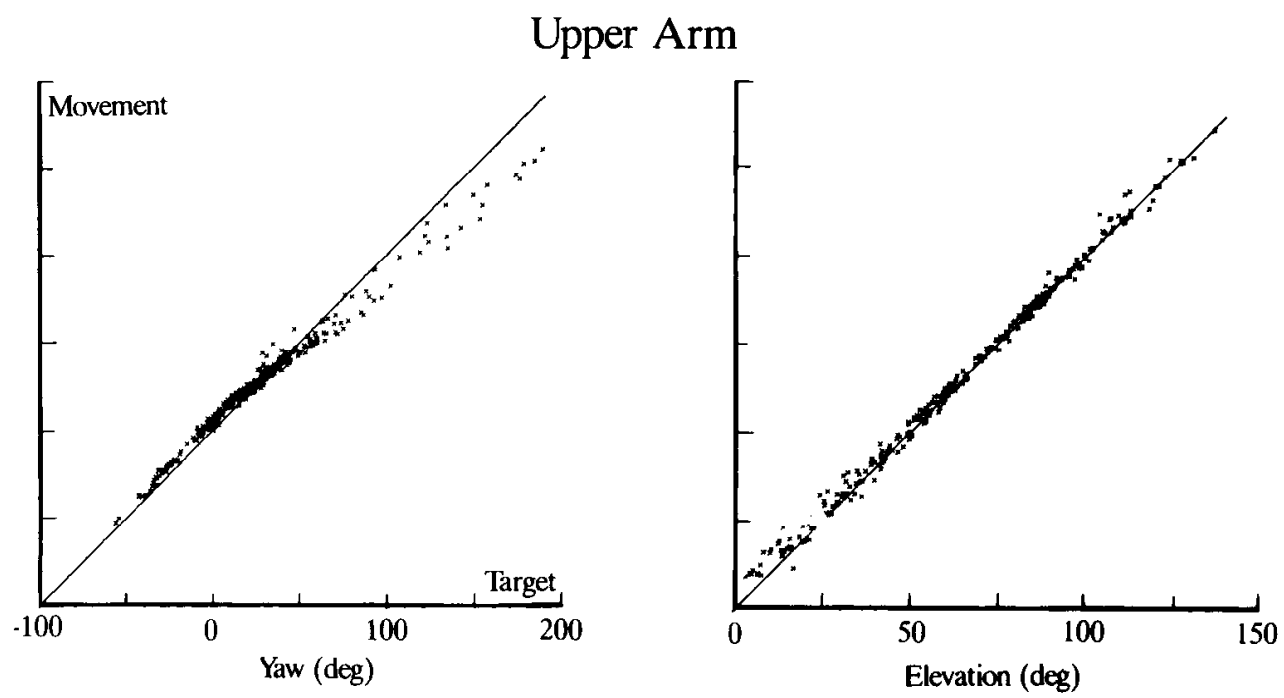

Lower Arm

Figure 4. Constant error for the MP condition represented in arm orientation coordinates for data pooled from 4 subjects. The data for the MP condition showed more scatter when represented in arm orientation coordinates than in end-point coordinates. Presentation format is as in Figure 3.
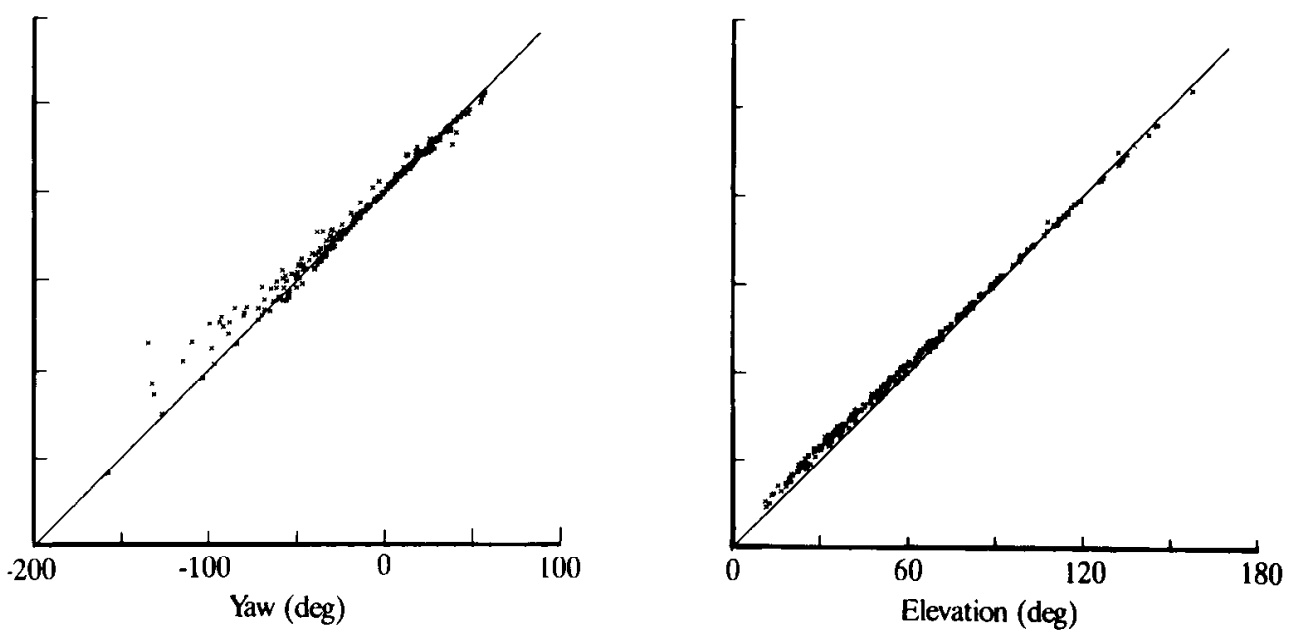

defining the locations chosen by the subjects showed a greater degree of dependence on the values of the other parameters defining arm orientation.

Because this task could be performed with 1 of 2 distinct strategies (match the end point or match the orientation angles), we compared the variable errors resulting from the fit to the end point to the variable errors resulting from the fit to the orientation angles. As shown in Figure 5, we found that the variable error in the segment orientation representation seemed to be larger than the error for the same condition in end-point coordinates.

In this figure, we show the variable error at the wrist instead of at the end point (for reasons to be explained shortly.) It can be seen by comparing Figure 5 with Figure 2, where the variable error is shown at the end point (fingertip), that the 2 measures give comparable results. The close similarity of these 2 measures also shows that the subjects used primarily their proximal joints to perform this task: Potential wrist movements did not appreciably affect the variable error.

The larger variable error in orientation angles together with the increased scatter shown in Figure 4 suggest the possibility that, in the passive matching condition, subjects attempted to match the end-point position rather than the orientation angle parameters. This might be possible if the subjects, for example, changed joint angles in a way that allowed them to put their index fingers at the target location using an arm configuration different from the configuration imposed by the passive displacement. Because of the complicated geometry of arm movements, however, it is difficult to predict the relationship between variability in orientation coordinates and variability in endpoint coordinates.

To determine whether the variability in the end point was less than the variability predicted by the segment orientation angles, we performed a simulation. For each of the 4 subjects, we took the passively displaced arm positions and used the bestfit equations of form (2) from that subject to generate a predicted set of orientation angles. To each of the simulated segment orientation parameters, we added random noise from a gaussian distribution with an SD equal to the variable error for that subject, parameter, and condition (i.e., we assumed that the 

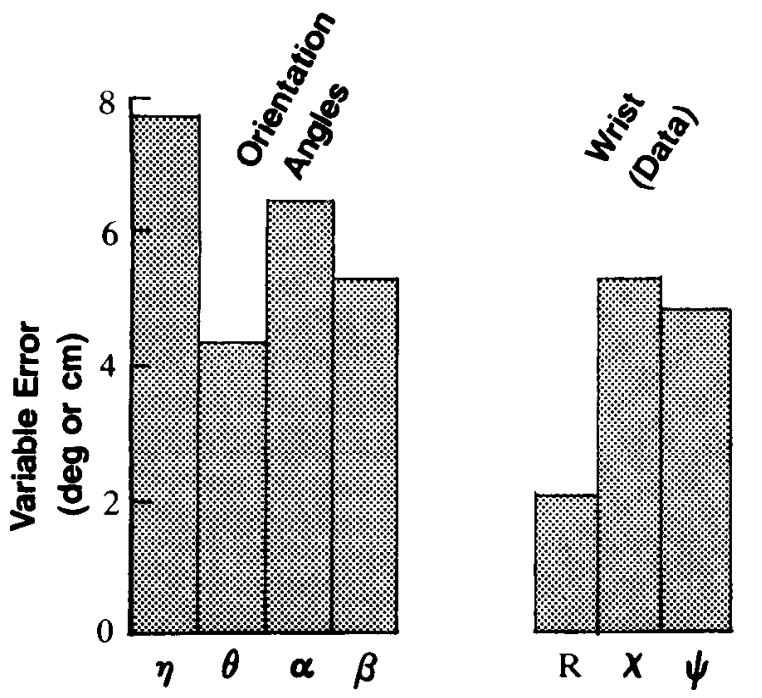

variable errors in the segment orientation angles were independent, contrary to what the case would be if the subjects were matching the end point). We also computed the lengths of the $2 \mathrm{arm}$ segments from the lucations of the shoulder, elbow, and wrist. From the new set of orientation angles with random noise added, we reconstructed a wrist position. Because our data did not include the angles of the wrist, we were unable to reconstruct the actual end point. Therefore, we compared the positions of the wrist from the MP data set with those predicted by the simulation. We made this comparison by performing regression analyses, with the end point defined as the wrist positions, for the data from both the MP condition and the simulation. These rcgressions gave, for each case (MP condition and simulated wrist positions), a set of equations relating the location of the chosen end point to the target end point. The variability in performance not accounted for in these 2 sets of analyses is shown in Figure 5. It can be seen that the variability in matching
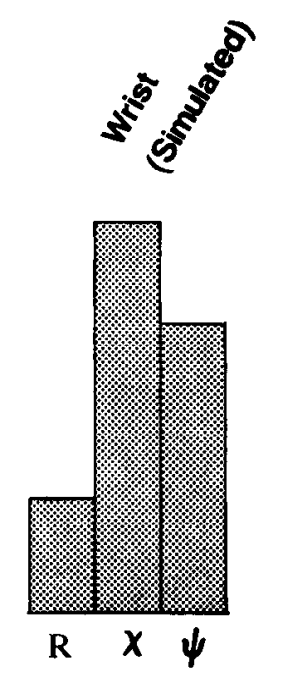

Figure 5. A simulation of variable error in end-point (wrist) coordinates. Variable error for the MP condition is represented in arm orientation coordinates and in end-point coordinates from both the data and the simulation of performance. Each bar represents the mean value from 4 subjects.

the arm segment orientation angles almost exactly accounted for the variability in the end-point position. Therefore, the performance of the subjects on this task can be explained by assuming that their strategy was one of matching the arm orientation angles of the passive displacement.

\section{Match end point (pointer, dark)}

As we have already shown, when subjects were asked to use the pointer to indicate the end point of an unseen passive movement, there was a significant increase in the variability of performance (Fig. 2). As shown in Figure 6, the constant error in indicating the end point was also large. Because of the scatter in the best-fit data, it is apparent that the location chosen by the subjects was not simply related to the target parameters (see Fig. 3 for comparison). In particular, the data for movement distance and elevation both show a dependence on other target parameters (vertical scatter in the plots). Thus, in addition to a

\section{Match Endpoint, Pointer}
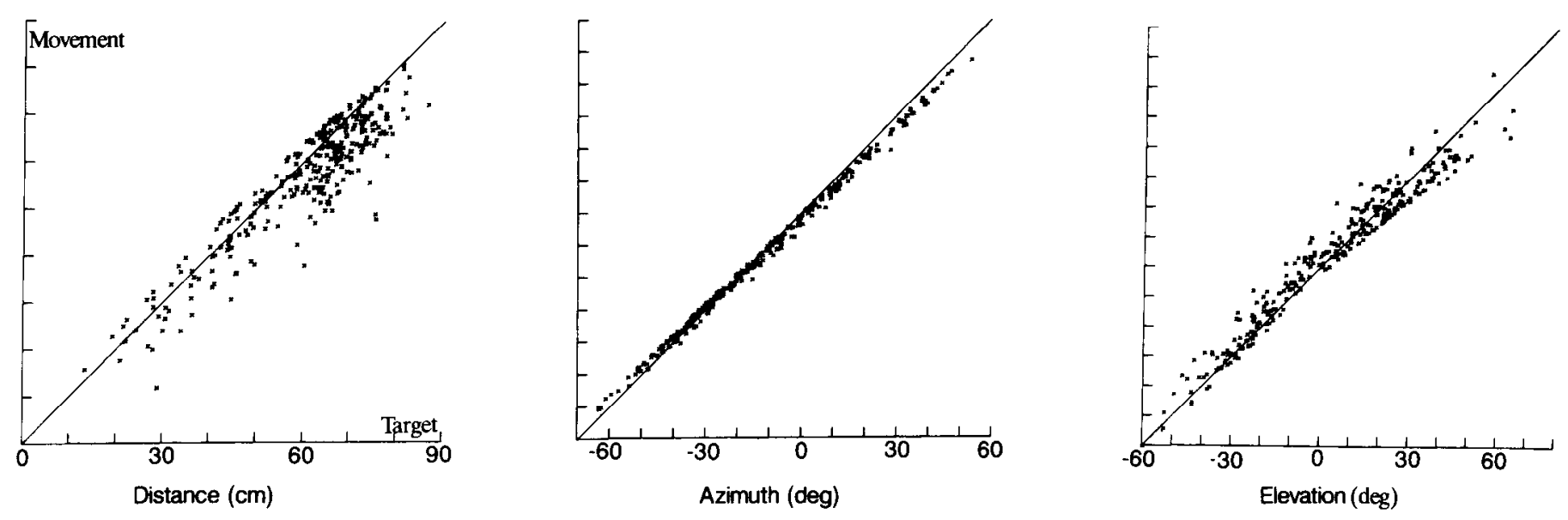

Figure 6. Constant error for the ME,dk condition for pooled data from 4 subjects. Estimates of the spatial locus of the hand from kinesthetic information showed both marked deviations from linearity and a strong dependence on the location in the work space. Presentation format is as in Figure 3. 
Virtual Target, Pointer
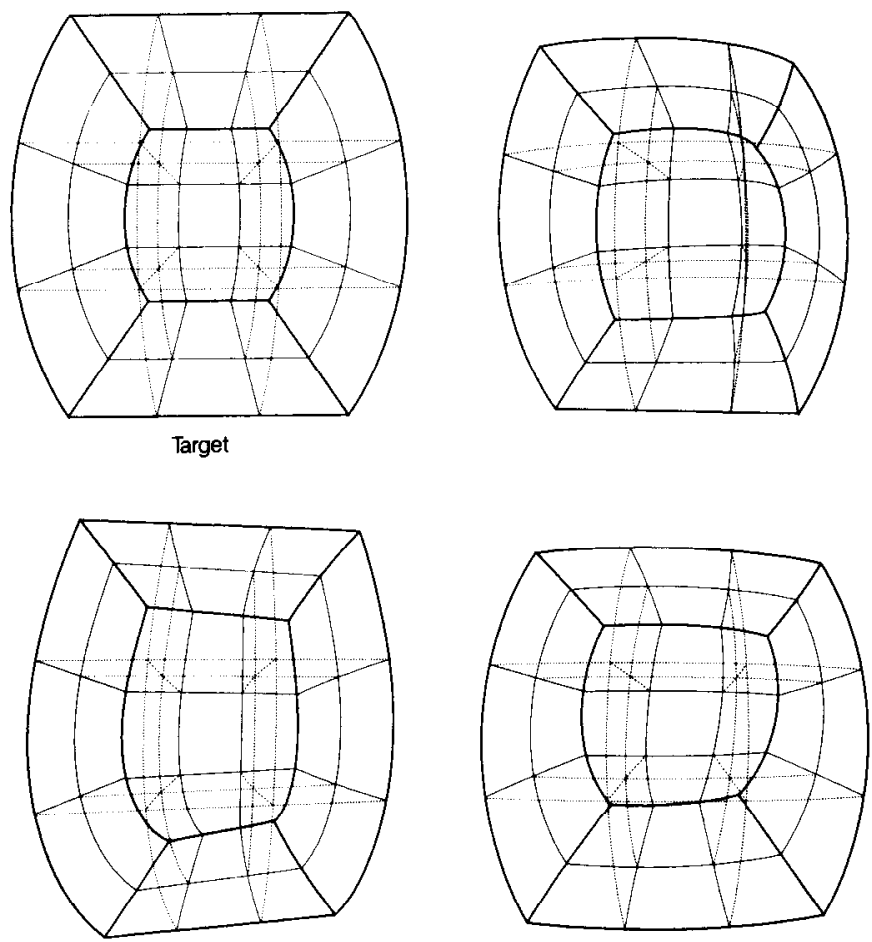

Figure 7. Perspective plots of constant error for 3 subjects in the VT condition. Performance in the VT task showed small constant error and high consistency between subjects. To the upper left is shown the representation of space in these plots for a perfect performance.

large amount of variability from trial to trial in each of the parameters specifying end-point location (Fig. 2), Figure 6 illustrates that the distance between the end point predicted by the best-fit equations and the target location was increased over MP $(8.37 \pm 1.17 \mathrm{~cm}$, mean \pm SE for 4 subjects $)$ and was dependent on the location of the target in the work space.

Furthermore, for the ME,dk condition, the performance in terms of constant error varied substantially among subjects in the performance of the task. To demonstrate the extent of this variability, we show in the perspective plots in Figures 7 and 8 the distribution throughout the work space of the end points chosen by the subjects. The grid to the upper left in Figure 7, labeled "Target," shows the way the target space is represented in these grids. The grid is a view from behind the right shoulder and forms that portion of a sphere that constitutes the subject's work space. Four vertical planes indicate constant azimuth at
Match Endpoint, Pointer
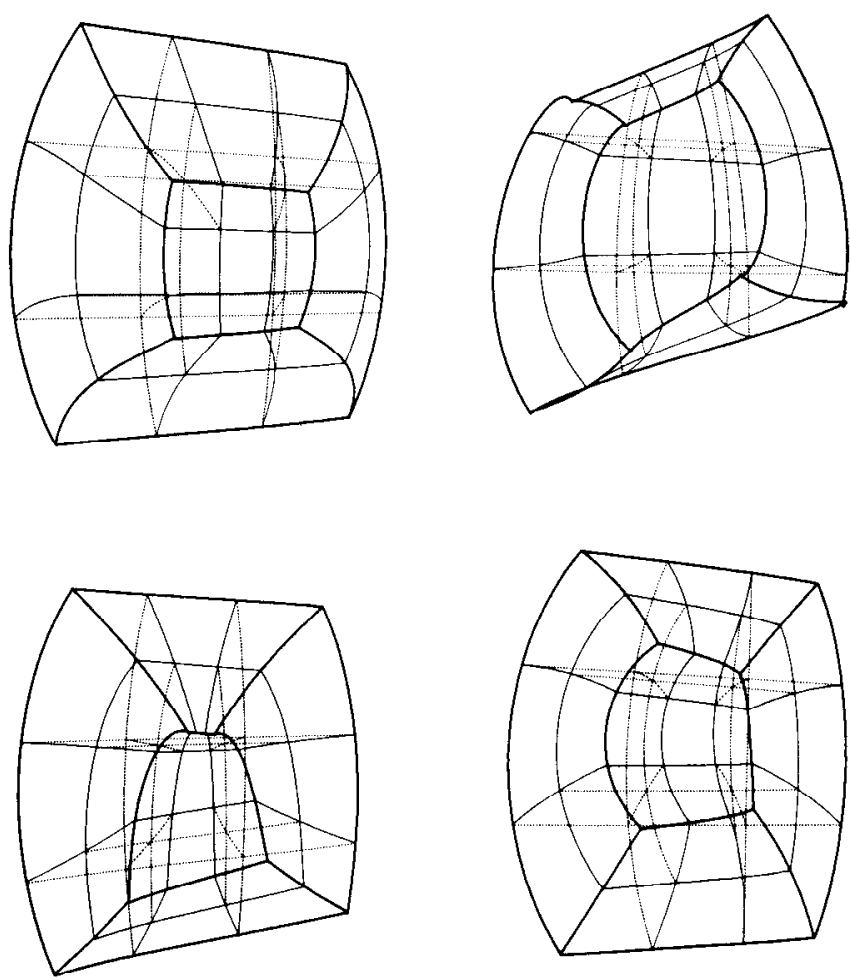

Figure 8. Perspective plots of constant error for 4 subjects in the $\mathrm{ME}, \mathrm{dk}$ condition. Performance in the ME, dk task resulted in large constant error and low consistency between subjects. Plots from the 3 subjects shown in Figure 7 are shown in corresponding panels in this figure.

$-45^{\circ},-15^{\circ}, 15^{\circ}$, and $45^{\circ}$ medial to lateral. Four planes perpendicular to the azimuth planes indicate constant elevation at the same angles, and 3 surfaces indicate constant distance at 30,50, and $70 \mathrm{~cm}$. The other grids in Figures 7 and 8 are constructed from the best-fit equations representing the performance of individual subjects. "Performance" grids from 3 representative subjects from the VT condition are shown in Figure 7. These 3 grids are presented as an example of a performance with low variability among subjects.

In Figure 8, we present the grids from the ME,dk condition. Three of the grids from the same subjects depicted in Figure 7 are presented at corresponding positions in Figure 8. Two aspects of these grids can be noted: First, there is a marked distortion in every grid. Second, the actual nature of the distortion

Table 1. Regression coefficients for the fit of chosen end point to passive displacement orientation parameters

\begin{tabular}{lrrllr}
\multicolumn{5}{l}{ Linear coefficients } \\
\cline { 2 - 6 } Parameter & \multicolumn{1}{l}{ Constant offset } & \multicolumn{1}{l}{$\eta$} & \multicolumn{1}{l}{$\alpha$} & \multicolumn{1}{l}{$\beta$} \\
\hline$R$ & $2.61 \pm 0.44$ & $-0.13 \pm 0.05$ & $0.37 \pm 0.05$ & $0.07 \pm 0.04$ & $0.23 \pm 0.03$ \\
$\chi$ & $-0.30 \pm 3.05$ & $0.27 \pm 0.17$ & $0.00 \pm 0.16$ & $0.46 \pm 0.13$ & $0.04 \pm 0.18$ \\
$\Psi$ & $-4.28 \pm 0.81$ & $-0.01 \pm 0.01$ & $0.24 \pm 0.03$ & $0.02 \pm 0.02$ & $-0.65 \pm 0.06$ \\
\hline
\end{tabular}

Data in this table are the mean of 4 subjects \pm SE. 
varies considerably among the 4 subjects. Each of the subjects adopted a different means of estimating the end-point location of their passivcly displaced arm.

It has previously been shown that pointing movements are accomplished by an approximation in which the orientation angles of the arm are linearly derived from target parameters (Soechting and Flanders, 1989b). It seemed possible that subjects would be able to invert the same transformation to derive the location of the hand in space from the orientation of the arm. The transformation described in Soechting and Flanders (1989b) provides a unique mapping from a 3-D space (target location in spherical coordinates) to a $4-\mathrm{D}$ space (the $4 \mathrm{arm}$ orientation angles). By including the constant offset coefficients from the original equations (cf. Soechting and Flanders, 1989b), the transformation matrix is converted from a $3 \times 4$ matrix into a $4 \times 4$ matrix that can be inverted. This addition results in a constraining equation, which is given as

$$
3.1 \eta_{T}-2.5 \alpha_{T}=100 \text {. }
$$

This equation can be solved for $\eta$ or $\alpha$ and the result substituted into the remaining equations. These steps lead to the following predicted coefficients for the inversion of the linear transformation:

$$
\begin{aligned}
& R_{M}=-18.9+0.7 \theta_{T}+0.4 \beta_{T}, \\
& \chi_{M}=0.6 \eta_{T}+0.5 \alpha_{T}, \\
& \psi_{M}=27.5+0.2 \theta_{T}-0.4 \beta_{T} .
\end{aligned}
$$

The actual coefficients derived from the experimental data are presented in Table 1. In this table, it can be seen that the intersubject variability in the linear fit was substantial, and that in several instances, the averaged coefficients are substantially different (by as much as a factor of 2) from those predicted by the inverse transformation.

Furthermore, subjects make errors in the distance of pointing movements, pointing consistently to locations closer than the actual target location (Soechting and Flanders, 1989a). If subjects used the inverse transformation in the ME, $\mathrm{dk}$ task, then they should indicate a spatial location farther from their shoulder than their actual hand location. Figure 6 shows that this was not the case. Once again, subjects did not point far enough. These findings suggest that the subjects did not perform this task by simply inverting the linear transformation described previously.

\section{Match end point (pointer, light)}

As shown in Figure 2, the variable errors for the last condition (ME,lt) were not significantly different from the errors for the VT condition. The mean distance between the end point predicted by the best-fit equations and the target locations, however, was intermediate to the ME,dk and the VT conditions $(6.77 \pm 0.49 \mathrm{~cm}$ averaged across the 4 subjects). Allowing the use of vision in this task improved the reliability of performance. The locations that were chosen by the subjects, however, were not as close to the target locations as the end points chosen when they used the pointer to indicate a target for which only visual information was given (VT condition).

\section{Discussion}

In order to determine the coordinate frame in which information from visual and kinesthetic systems can be combined, we asked subjects to perform 1 of 2 movements to indicate the location of their hands following an unseen passive displacement. When we asked subjects to use a movement of the arm that had been passively displaced, the subjects were able to reliably indicate the location of their hands. When we asked the subjects to use a pointer to indicate the spatial location of their hands following the passive displacement, however, the subjects were unable to reliably indicate this location. We showed that the errors in performance in the latter task were not simply due to the use of a pointer to indicate a spatial target. The subjects could use the pointer to indicate a visually specified target as well as they could use their arms to indicate a visually specified target. Nor were the errors due to a lack of kinesthetic information, as shown in the MP condition. Instead, the results suggest that the errors in performance occurred because subjects had difficulties in using kinesthetic information to synthesize a spatial representation of the hand's location.

While the MP experiment showed that subjects were able to make good use of kinesthetic information to position their arms in space, the experimental design contained an ambiguity. In performing this task, the subjects could have been simply matching the spatial orientation of their upper arms and forearms, or they could have been more attentive to the location of their hands following the passive displacement. Simulations showed that the performance of subjects on this task could be readily accounted for by assuming that the subjects matched the arm orientation angles of the passive displacement.

These data suggest that kinesthetic information is most readily utilized when it is represented in arm orientation parameters. This is consistent with the model described in the introductory remarks. The model suggests that, when kinesthetic information is used in the computation of movement parameters, the computations are performed in a coordinate system based on arm orientation parameters (Soechting and Flanders, 1990; Flanders, Helms Tillery, and Soechting, unpublished observations). This lends support to the interpretation of Soechting and Flanders $(1989 a, b)$ that subjects transformed a visual representation of target location into a representation of the arm segment orientations that would place the hand near the target. The suggestion is that those transformations are performed in order to represent visually derived information in a kinesthetic reference frame. Once the visually and kinesthetically derived information is represented in this common frame, the motor error signal that will give rise to an arm movement can be computed.

While the model aids in the interpretation of a number of psychophysical and neurophysiological results, we do not intend to suggest that each of the representations and computations described are comprised of discrete entities in the CNS. Instead, we view the representations as occurring on a continuum from a visual reference frame to a kinesthetic reference frame. Furthermore, we believe that information derived from each of the sensory modalities interacts at a number of levels and can have an impact on computations that, a priori, require input from no more than a single sensory modality. An interaction of visual and kinesthetic information is illustrated by the results of the final experiment.

When we allowed subjects to see the passive displacement of their arms and then asked them to indicate with the pointer the location of their hands, the variability in their performance decreased to levels comparable to the VT condition. The mean distance between the target location and the location predicted by the best-fit equations, however, did not return to the values found in the VT condition. When vision of the passively dis- 
placed arm was available, the reliability of performance increased, but the locations chosen were still in error. This indicates that, cven though kinesthetic information does not provide a good estimate of the hand's spatial location, it nonetheless is not discarded by the CNS when it is available.

This type of interaction between sensory modalities has been shown for a number of systems. For example, it has been shown that visual information affects the processing of inputs from both auditory (Held, 1955; Kalil and Freedman, 1967; Knudsen and Knudsen, 1985; Jay and Sparks, 1987) and somatosensory (Harris, 1965; Craske, 1975; Posner et al., 1976; Lackner, 1981) modalities. It may, in part, serve to calibrate those systems for spatial localization (McCloskey, 1978; Knudsen and Knudsen, 1989) and can alter perception derived from those modalities. Our last finding suggests, conversely, that kinesthetic information also affects the visual representation of a spatial location, even though the information provided by the kinesthetic system is not reliable. Therefore, while the model provides a framework in which to think about the processes involved in this task, it cannot be assumed that the modules and computations described are independent entities.

\section{References}

Craske B (1975) A current view of the processes and mechanisms of prism adaptation. In: Aspects of neural plasticity (Vital-Durand F, Jeannerod M, eds), pp 125-138. Paris: Editions de l'institut national de la santé et de la recherche medicale.

Flanders M, Soechting JF (1990) Parcellation of sensorimotor transformations for arm movements. J Neurosci 10:2420-2427.

Georgopoulos AP, Kettner RE, Schwartz AB (1988) Primate motor cortex and free arm movements in three-dimensional space. II. Coding of the dircetion of movement by a neuronal population. J Neurosci 8:2928-2937.

Harris CS (1965) Perceptual adaptation to inverted, reversed, and displaced vision. Psychol Rev 72:419-444.

Held R (1955) Shifts in binaural localization after prolonged exposures to atypical combinations of stimuli. Am J Psychol 68:526-548.
Jay MF, Sparks DL (1987) Sensorimotor integration in the primate superior colliculus. II. Coordinates of auditory signals. J Neurophysiol $57: 35-55$.

Johnson RA, Wichern DW (1982) Applied multivariate statistical analysis, pp 291-358. Englewood Cliffs, NJ: Prentice Hall.

Kalil R, Freedman SJ (1967) Compensation for auditory rearrangement in the absence of observer movement. Percept Mot Skills 24: $475-478$

Knudsen EI, Knudsen PF (1985) Vision guides the adjustment of auditory localization in young barn owls. Science 230:545-548.

Knudsen EI, Knudsen PF (1989) Vision calibrates sound localization in developing barn owls. J Neurosci 9:3306-3313.

Lackner JR (1981) Some aspects of sensory-motor control and adaptation in man. In: Intersensory perception and sensory integration (Walk RD, Pisk HL, eds), pp 143-173. New York: Plenum.

McCloskey DI (1978) Kinesthetic sensibility. Physiol Rev 58:753820.

Paillard J, Brouchon M (1968) Active and passive movements in the calibration of position sense. In: The neuropsychology of spatially oriented hehavior (Freedman SJ, ed), pp 37-55. Homewood, II : Dorsey.

Posner MI, Nissen MJ, Klein RM (1976) Visual dominance: an information-processing account of its origins and significance. Psychol Rev 83:157-171.

Press WH, Flannery BP, Teukolsky SA, Vetterling WT (1988) Numerical recipes in C: the art of scientific computing, pp 517-557. Cambridge: Cambridge UP.

Soechting JF, Flanders M (1989a) Sensorimotor representations for pointing to targets in three-dimensional space. J Neurophysiol 62: 582-594.

Soechting JF, Flanders M (1989b) Errors in pointing are due to approximations in sensorimotor transformations. J Neurophysiol 62: 595-608.

Soechting JF, Flanders M (1990) Deducing central algorithms of arm movement control from kinematics. In: Motor control: concepts and issues (Humphrey DR, Freund H-J, eds), pp 293-306. Wiley.

Soechting JF, Ross B (1984) Psychophysical determination of coordinate representation of human arm orientation. Neuroscience 13: 595-604.

Soechting JF, Tillery SIH, Flanders M (1990) Transformation from head- to shoulder-centered representation of target direction in arm movements. J Cogn Neurosci 2:32-43. 